\title{
CINESITERAPIA RESPIRATÓRIA NO DOENTE CRÍTICO COM COVID-19: A INTERVENÇÃO DO ENFERMEIRO DE REABILITAÇÃO - ESTUDO DE CASO
}

\section{KINESITERAPIA RESPIRATORIA EN EL PACIENTE CRÍTICO CON COVID-19: INTERVENCIÓN DE ENFERMERA DE REHABILITACIÓN - ESTUDIO DE CASO \\ RESPIRATORY KINESITHERAPY IN CRITICALLY ILL PATIENTS WITH COVID-19: THE REHABILITATION NURSE'S INTERVENTION - CASE STUDY}

DOI 10.33194/rper.2020.v3.s2.8.5796 | Submetido 24/07/2020 | Aprovado 16/11/2020

\begin{abstract}
Marco Fernando Neves China ${ }^{1}$; Helena Isabel Bacalhau Antunes ${ }^{2}$; Lúcia Maria Simões Martins 1 ; Maria de Fátima Alves Pereira Ferreira ${ }^{2}$; Maria Fernanda Jesus Simões Viseu1 ${ }^{1}$; Maria Helena Dias Pires ${ }^{2}$

1 - Centro Hospitalar Universitário de Coimbra - Pólo HG; 2 - Centro Hospitalar Universitário de Coimbra - Pólo HUC
\end{abstract}

\section{RESUMO}

Objetivo: Este estudo tem como objetivo: descrever as intervenções do enfermeiro de reabilitação em termos de Cinesiterapia Respiratória na pessoa com COVID-19, com ventilação mecânica e em oxigenação extra-corpórea por membrana, analisando a resposta em termos de parâmetros gasométricos e ventilatórios antes e após cada sessão.

Método: Estudo de caso de uma pessoa com 63 anos, com antecedentes de obesidade e hipertensão arterial. Realizouse colheita de dados retrospetiva de processo clínico, referente a um período de internamento em $\mathrm{UCl}$ de 38 dias.

Resultados: As técnicas mais utilizadas na Cinesiterapia Respiratória foram a aspiração de secreções em circuito fechado, vibrações, descompressão brusca, aberturas costais seletivas, expiração manual forçada, ventilação dirigida e in-exsuflador mecânico. Na globalidade das sessões, verificou-se uma melhoria nos volumes inspiratórios e expiratórios, assim como melhoria na compliance estática. Começou a usar-se o in-exsuflador mecânico ao fim de uma semana de internamento, depois de se esgotarem todas as intervenções possíveis, sendo que todos os outcomes apresentaram melhorias globais, sem eventos de adversos.

Conclusão: A implementação de intervenções de cinesiterapia respiratória estruturadas e individualizadas, podem contribuir significativamente na recuperação da pessoa em situação crítica, tendo-se usado com eficácia técnicas que não estavam descritas para casos COVID- 19.

Palavras-chave: Cinesiterapia Respiratória; COVID-19; ventilação mecânica; Enfermeiro Reabilitação; ECMO-VV; inexsuflador mecânico

\section{RESUMEN}

Objetivo: Este estudio tiene como objetivo: describir las intervenciones del enfermero de rehabilitación en términos de Kinesiterapia Respiratoria en los pacientes con COVID-19 sobre ventilación mecánica y oxigenación com membrana extracorpórea, analizando la respuesta en términos de parámetros gasométricos y ventilatorios antes y después de cada sesión.

Método: Estudio de caso de un paciente de 63 años con antecedentes patológicos de obesidad y hipertensión arterial. Se recopilaron datos retrospectivos del proceso clínico, durante una permanência en UCl de 38 días.

Resultados: Las técnicas más utilizadas en Kinesiterapia Respiratoria fueron, la aspiración de secreciones en circuito cerrado, vibraciones, descompresión repentina, aberturas costales selectivas, espiración manual forzada, ventilación dirigida y in-exsuflador mecánico. En todas las sesiones, hubo una mejoria en los volúmenes inspiratorio y espiratorio, así como en la compliance estática. El in-exsuflador mecánico se inició después de una semana de hospitalización, después de agotarse todas las intervenciones posibles, siendo que todos los resultados mostraron mejoras globales, sin eventos adversos.

Conclusión: La implementación de intervenciones de kinesiterapia respiratoria estructuradas e individualizadas puede contribuir significativamente en la recuperación de un paciente en una situación crítica, habiendo sido utilizadas técnicas que aín no se han descrito para los casos de COVID-19 de manera efectiva.

Palavras clave: Kinesiterapia Respiratoria; COVID-19; Ventilacion mecanica; Enfermero de rehabilitación; ECMO; inexsuflador mecánico 
Aim: This study aims to describe rehabilitation nurse interventions in Respiratory Kinesitherapy in critically ill with COVID-19 patient undergoing venovenous extra-corporeal membrane oxygenation and its response concerning to gasometric and ventilatory parameters before and after each session.

Methodology: Case study of a 63-year-old person with history of obesity and high blood pressure. Retrospective data were collected from the clinical process, referring to a 38-day ICU stay.

Results: The most performed techniques used in Respiratory Kinesitherapy by rehabilitation nurses were suctioning through closed system, vibrations, sudden decompression, selective costal openings, forced manual expiration, directed ventilation and mechanical in-exhaler. Globally, all sessions showed that there was an improvement in inspiratory and expiratory volumes, as well as in static compliance. Machanical in-exhaler was used after a week of hospital stay, after all other possible interventions were performed, and all outcomes showed global improvements, without any adverse events.

Conclusion: Implementation of structured and individualized respiratory kinesitherapy interventions can significantly benefit recovery of critical ill patients. Techniques that weren't described for covid 19 cases were used effectively.

Keywords: Respiratory Kinesitherapy; COVID-19; mechanical ventilation; Rehabilitation Nurse; ECMO-VV; mechanical in-exhaler

\section{INTRODUÇÃO}

A doença Coronavírus 2019 (COVID-19) é a expressão clínica da infeção pelo vírus zoonótico SARS-CoV-2. Esta doença apresenta-se na maioria dos casos $(80 \%)$, em formas ligeiras a moderadas, contudo $6,1 \%$ terão doença crítica com necessidade de internamento numa Unidade de Cuidados Intensivos (UCI) ${ }^{(1)}$.

Os motivos mais comuns para a admissão em UCl são a insuficiência respiratória hipoxémica e hipotensão que requerem ventilação mecânica invasiva (VMI) e tratamento vasopressor respetivamente. A maioria dos doentes admitidos em $\mathrm{UCl}$ apresenta falência multiorgânica, incluindo síndrome de dificuldade respiratória aguda (SDRA). Para doentes com SDRA, os internamentos prolongados em $\mathrm{UCl}$ têm um impacto significativo na redução da função pulmonar e na atividade muscular ${ }^{(2)}$.

Ensaios clínicos em rede demonstraram que uma estratégia de ventilação mecânica protetora, baseada em volumes correntes baixos, foi capaz de reduzir a mortalidade por SDRA. Se os níveis de oxigenação permanecerem baixos com altos níveis de pressão positiva no final da expiração (PEEP), outros procedimentos avançados podem ser realizados: posição prono (PP) e a oxigenação extra-corpórea por membrana veno-venoso (ECMO- VV) ${ }^{(3)}$.

A ECMO-VV é um tratamento que atua essencialmente substituindo os pulmões por um oxigenador artificial externo para executar as trocas gasosas. Apesar de eficaz, a ECMO-VV não está isenta de riscos, nomeadamente devido à colocação percutânea de cânulas de grande calibre, necessidade de anticoagulação sistémica, acarretando risco de eventos hemorrágicos-trombóticos ${ }^{(3)}$. Durante a intervenção do Enfermeiro Especialista de Enfermagem de Reabilitação (EEER) é importante a vigilância de hemorragias nos locais de inserção dos acessos vasculares, do reflexo pupilar e das características das secreções, entre outras.

As intervenções do EEER em contexto de doença crítica, em $\mathrm{UCl}$, visam a redução da incidência de fraqueza muscular adquirida e melhoria da qualidade de vida e prognóstico vital dos doentes. No entanto, não existe prática descrita nem evidência em contexto COVID-19, o que acarreta que as intervenções de reabilitação devam ser avaliadas individualmente e de forma dinâmica, adaptando-se às mudanças rápidas que caraterizam a progressão da doença ${ }^{(1)}$.

Após a vivência de uma situação que exigiu da equipa de saúde, designadamente dos EEER, uma série de decisões difíceis, considerou-se útil partilhar as mesmas. Assim foram definidos como objetivos: descrever as intervenções em termos de Cinesiterapia Respiratória (CR) na pessoa com COVID-19 em ECMOVV, analisando a resposta em termos de parâmetros gasométricos e ventilatórios.

\section{METODOLOGIA}

Seguiu-se a metodologia de estudo de caso, de acordo com as indicações de Budgell ${ }^{(4)}$.

Os cuidados de Enfermagem de Reabilitação na CR foram efetuados diariamente e em alguns dias foram bidiários. Decorreram entre os meses de abril e maio, numa UCl de um Centro Hospitalar da Administração Regional de Saúde do Centro.

A colheita de dados foi realizada mediante anamnese e consulta do processo clínico, referente ao período em que o doente esteve em VMI (entre o D2 e D38), tendo sido realizada de forma completamente anonimizada, garantindo a irreversibilidade da mesma. Foi igualmente obtido o consentimento informado do familiar da doente.

Considerou-se como outcomes de eficácia: os valores gasométricos ( $\mathrm{FiO2}, \mathrm{PaO} 2, \mathrm{PaCO} 2$ e $\mathrm{SO} 2)$ avaliados por gasómetro RAPIDPoint $\AA \quad 500$; os parâmetros ventilatórios (volume inspiratório, volume expiratório, compliance estática) avaliados no ventilador Servo I e a evolução radiológica (Radiografia no leito, anterior). $\mathrm{Na}$ análise dos dados determinou-se a diferença após-antes $\mathrm{CR}$, em termos de valores gasométricos e ventilatórios. 


\section{Evolução do estado clínico do doente}

Doente de 63 anos, sexo feminino, raça caucasiana, e nacionalidade portuguesa. Profissão comerciante e reside com o marido, previamente autónoma nas atividades de vida diária. Apresenta como antecedentes pessoais: obesidade e hipertensão arterial.

A doente iniciou sintomas de febre $\left(38,0-38,5^{\circ} \mathrm{C}\right)$, mialgias, tosse seca e diarreia, vindo a agravar a situação, recorreu ao serviço de urgência com 7 dias de evolução. A radiografia $(R X)$ tórax evidenciava infiltrados bilaterais e a tomografia axial computorizada torácica revelou "infiltrados parenquimatosos bilaterais com densidade vidro despolido de distribuição predominantemente periférica a toda a altura dos campos pulmonares, compatível com infeção vírica por COVID-19". Por agravamento das trocas gasosas e radiológico e com necessidade crescente de $\mathrm{FiO2}$, foi realizada entubação endotraqueal e a doente foi transferida para UCI para VMI e submetida a PP.

Parâmetros ventilatórios com Fracção Inspirada de Oxigénio ( $\mathrm{FiO2})$ de $100 \%$, PEEP $14 \mathrm{cmH} 20$, relação $\mathrm{PaO} 2 / \mathrm{FiO} 2$ <100 e com valores de gasometria: $\mathrm{PaCO} 2$ $53 \mathrm{mmHg}, \mathrm{PaO} 2$ 102mmHg, $\mathrm{HCO} 329.7$, lactatos $1.6 \mathrm{e} \mathrm{Ph}$ 7,34. Foi referenciada para ECMO-VV, sendo realizada canulação veno-venosa e resgatada pela equipa de ECMO desta UCI.

No D2 iniciou CR e apresentava $R X$ com opacidade bilateral (Imagem 1A). Durante o internamento a doente foi submetido a ventilação protetora.
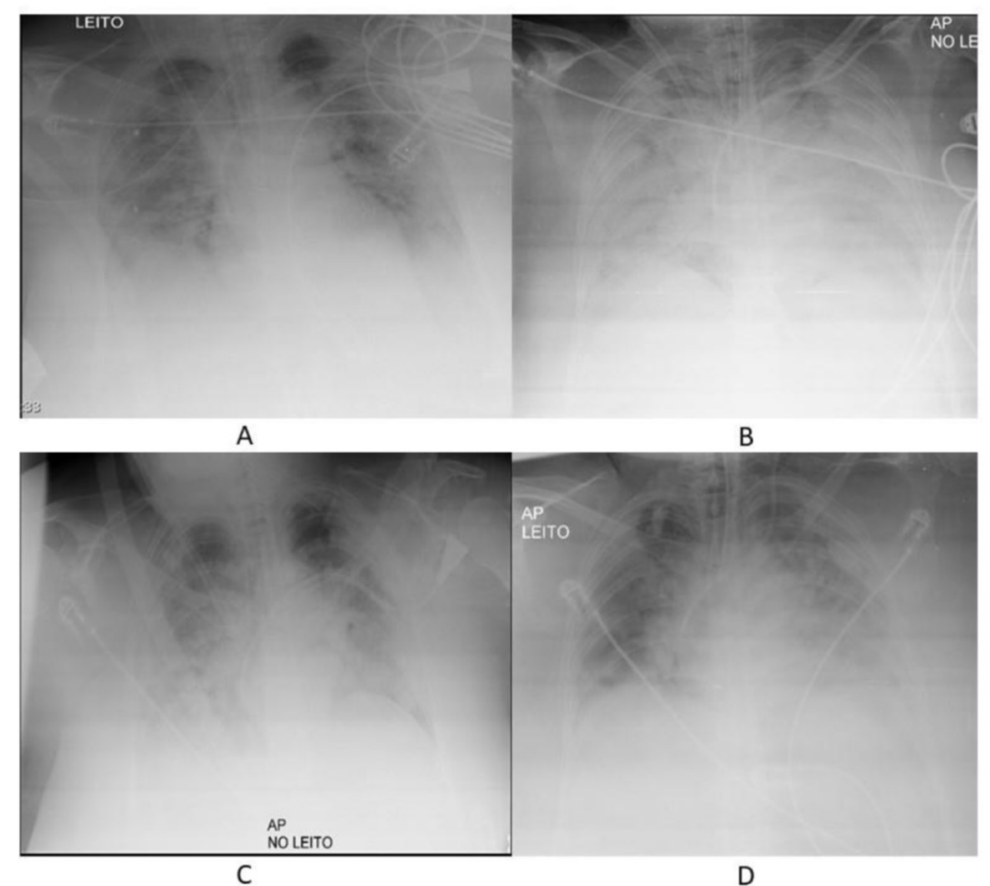

B

Imagem 1A- RX no D1: infiltrados bilaterais parenquimatosos com densidade em vidro despolido. Imagem $1 B-R X$ no $D 9$ (antes da CR e da utilização de I-EM): opacidades homogéneas bilaterais, apicocaudais, com broncograma aéreo. Imagem 1C- RX no D11 (dia após inicio l-EM): redução da extensão das opacidades descritas anteriormente. Diminuição das opacidades nos ápices, principalmente no esquerdo. Imagem 1D - RX no D24: opacidade homogénea ocupando $2 / 3$ do campo pulmonar esquerdo, com broncograma aéreo. À direita em menor extensão, em posição parahilar.
Ao D7 iniciou PP com melhoria das trocas gasosas e discreto aumento do volume corrente e de compliance apresentando drenagem de secreções em abundante quantidade.

Ao D9, foi associado às técnicas de CR a utilização do in-exsuflador mecânico (I-EM).

No D19 a doente foi decanulada do ECMO-VV, permanecendo em VMI (Pressão Controlada, FiO2 50\%, PEEP $8 \mathrm{cmH} 2 \mathrm{O}$ e Volume Total de $320 \mathrm{ml}$ ).

Ao $D 25$ realizou o último $P P$, foi traqueotomizada $\mathrm{e}$ suspensa a curarização no dia seguinte. Ao D30 suspendeu sedação.

Iniciou desmame ventilatório ao D31, com o primeiro treino de ventilação espontânea (VE) de 5 horas.

Ao D32 de internamento fez a primeira transferência para cadeirão, enquanto esteve sentada foi realizada a primeira video-chamada com o familiar de referência, que no decorrer do internamento manteve contacto diário. Foram realizadas transferências para o cadeirão sempre que a situação clínica o permitia e a partir do D38 ficou em VE definitiva com Oxigénio a $1 \mathrm{~L} / \mathrm{min}$.

\section{RESULTADOS}

Os resultados mostram que a CR teve início ao D2, onde foram realizadas 47 sessões (22 foram bi-diárias), mantendo as intervenções de CR até ao momento da alta. Ao D9 foi associado às técnicas de CR a utilização do I-EM, sendo este utilizado em 17 sessões.

Em análise ao processo de Enfermagem de Reabilitação identificaram-se dois diagnósticos de enfermagem relacionados com o foco respiração: a limpeza das vias aéreas ineficaz e ventilação comprometida. Relativamente à limpeza das vias aéreas e à ventilação as técnicas aplicadas encontram-se representadas no Gráfico 1.

Relativamente aos resultados dos outcomes dos valores gasométricos, verificou-se que na maioria das intervenções de $\mathrm{CR}$, a $\mathrm{PaO} 2$ e a $\mathrm{SO} 2$ diminuíram, a $\mathrm{PaCO} 2$ aumentou e na relação $\mathrm{PaO} 2 / \mathrm{FiO}_{2}$ houve igual número de tendências positivas e negativas. Por outro lado, na maioria das sessões os volumes inspiratórios e expiratórios, assim como a compliance estática aumentaram após a CR (Gráfico 2).

Analisando agora as sessões de CR em que foi utilizado - I-EM, todos os outcomes apresentaram melhorias globais, salientando-se nomeadamente, o volume inspiratório, a compliance estática, o $\mathrm{PaO} 2$ e a relação $\mathrm{PaO} 2 / \mathrm{FiO} 2$ com maiores percentagens de melhoria após a sessão (Gráfico 3).

Os resultados, nos dias em que o doente estava submetido a ECMO-VV e na CR foi utilizado I-EM, mostraram que todos os outcomes aumentaram, à exceção da $\mathrm{PaCO} 2$ que diminuiu ligeiramente (Gráfico 4).

Da observação do $R X$ tórax, verifica-se que existiu uma melhoria acentuada entre o D9 (Imagem 1B) e o D11 (Imagem 1C). O I-EM foi iniciado no D10, complementando as técnicas de CR. 
Exercício de tonificação Intercostal

Exercício de tonificação Diafragmática

Ventilação dirigida

Expiração forçada manual

Descompressão brusca

Aberturas costais seletivas (esq ${ }^{\mathrm{a}}, \mathrm{dt}^{\mathrm{a}}$ )

Cough assist

Drenagem postural modificada

Vibrações

Percussões

Aspiração de secreções
12

17

51

80

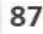

80

36

34

Acrónimos: $\mathrm{CR}$ - cinesiterapia respiratória; ECMO-VV - venovenous extracorporeal membrane oxygenation; I-EM - in-exsuflador mecânico

Gráfico 1 - \% Técnicas utilizadas na CR

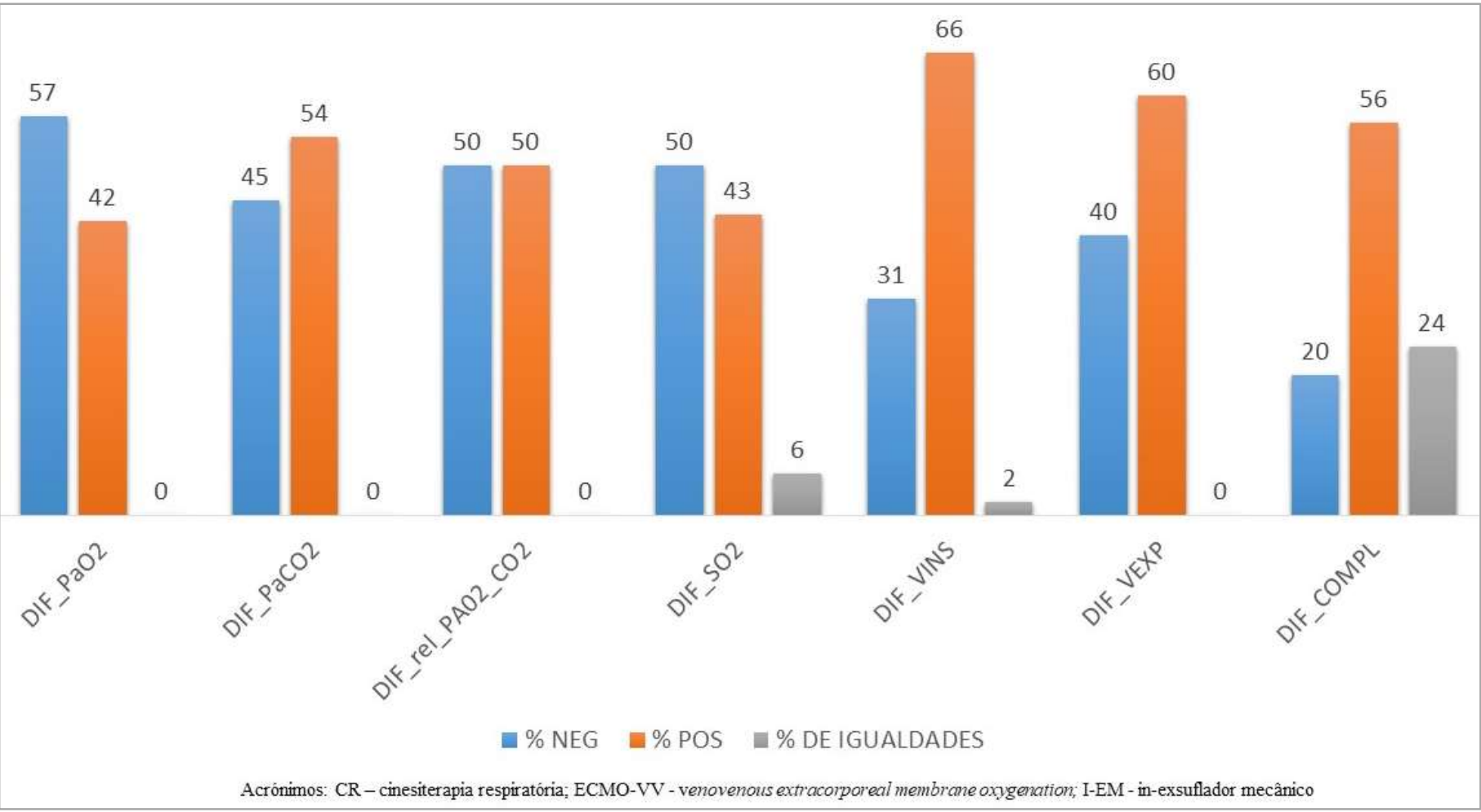

Gráfico 2 - \% Tendências positivas e negativas da $C R$ 


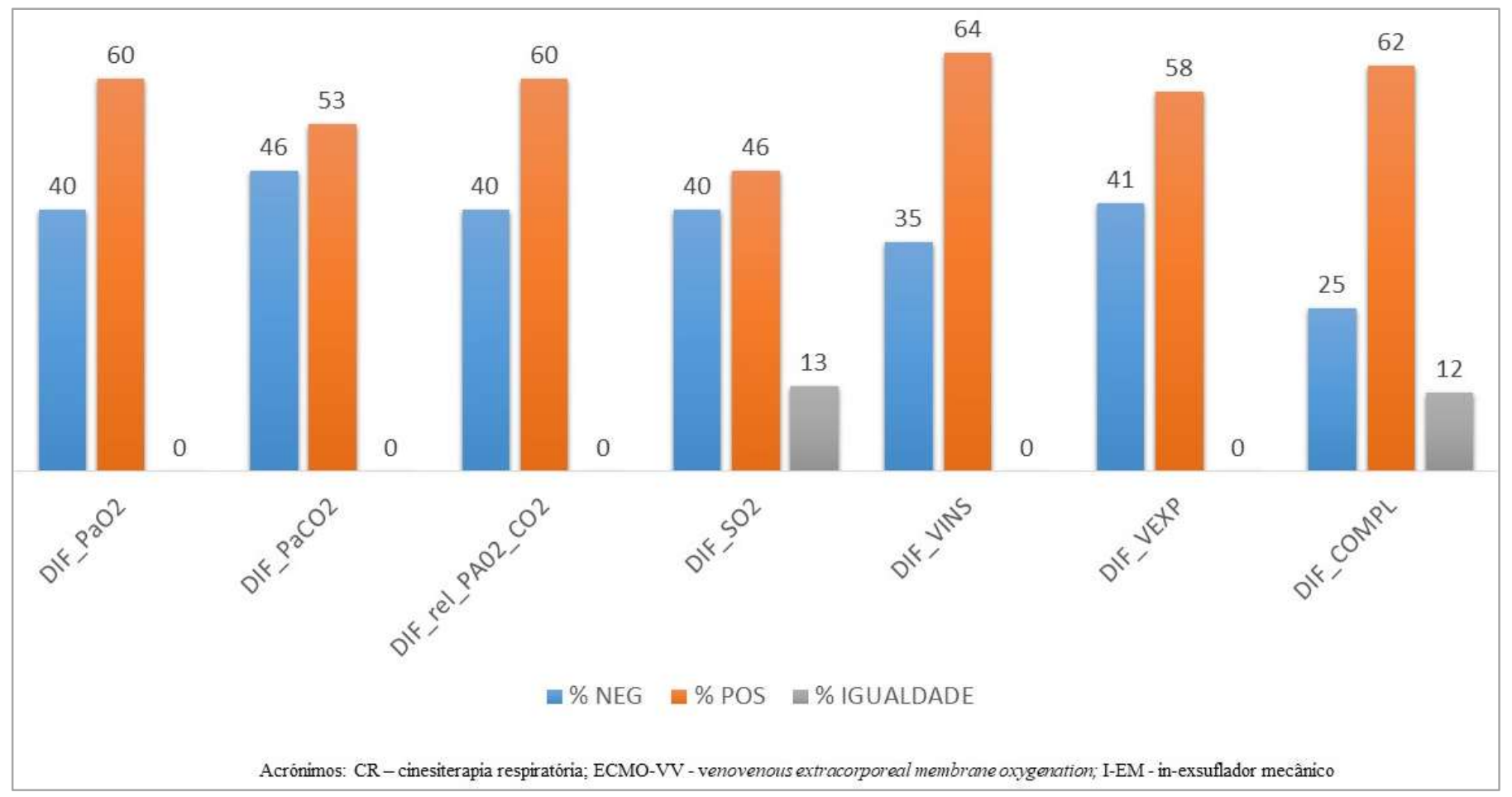

Gráfico 3 - \% Tendências positivas e negativas da CR com I-EM

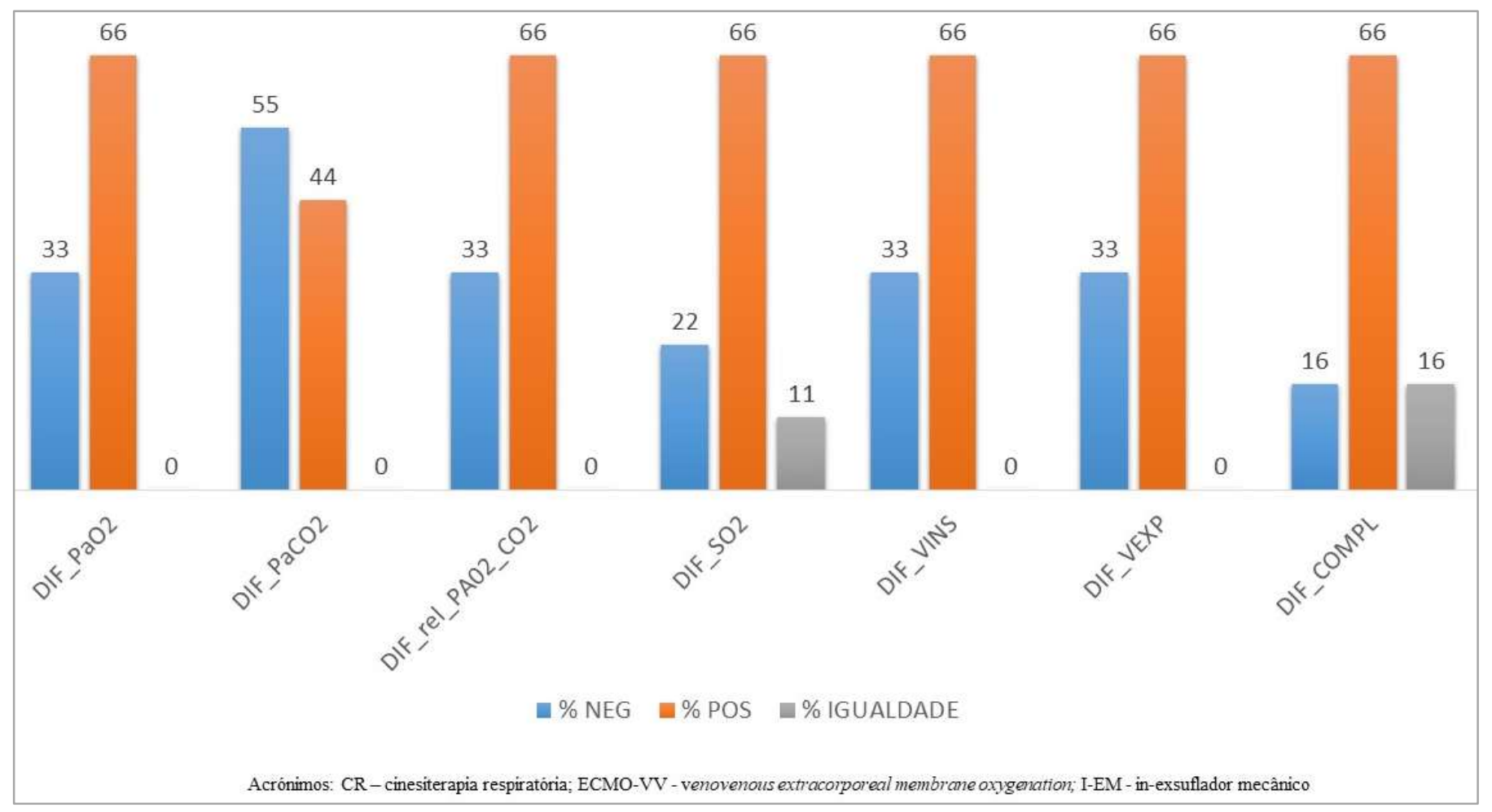

Gráfico 4 - \% Tendências positivas e negativas no doente com ECMO-VV e CR com I-EM

\section{DISCUSSÃO}

A evolução apresentada pela doente em estudo foi lenta e gradual devido à gravidade da doença e às técnicas invasivas a que foi sujeita. 0 que condicionou um longo período de sedação, curarização e analgesia levando a uma VMI prolongada e consequentemente, um desmame ventilatório difícil.

A intervenção prematura de reabilitação respiratória não é recomendada para doentes graves COVID-19, com critérios clínicos de instabilidade ou de deterioração progressiva por evolução da doença ${ }^{(1)}$. Neste caso, os
EEER após avaliação diária e criteriosa da doente em parceria com a equipa multidisciplinar, instituíram a CR precocemente (sem eventos adversos). Os resultados evidenciam que os cuidados diários de $C R$, complementados pelo I-EM, tiveram um impacto positivo na evolução clínica da doente.

0 estado da arte tem demonstrado que a implementação de $C R$ precoce na pessoa em situação crítica submetida a VMI, é segura e exequível com inúmeros benefícios: em termos funcionais, psicológicos e ventilatórios ${ }^{(5)}(6)$. 
Sendo um estudo num contexto de COVID-19, é pertinente referir as limitações que foram sentidas pelo ER: o uso de equipamento de proteção individual, que toldavam a perceção sensorial (as vozes abafadas, a capacidade auditiva diminuída e a visão dificultada pelo embaciamento dos óculos ou viseira); a impossibilidade de auscultar o doente; o risco de desconexões acidentais do circuito ventilatório; a gravidade do quadro clínico; a execução de determinadas técnicas que potenciassem o risco de propagação de aerossóis e a impossibilidade de estabelecer uma comunicação próxima com o doente.

As pessoas com COVID-19 têm alterações fisiopatológicas graves do interstício pulmonar (aumento da permeabilidade da membrana alvéolocapilar; alteração da perfusão pulmonar decorrente de shunt intrapulmonar), com colapso alveolar (predomínio na região dorsal), redução da compliance pulmonar, entre outras alterações ${ }^{(7,8)}$. A CR nestes doentes ventilados deve ser adaptada ao risco de contaminação e propagação do vírus, de modo a evitar a aerossolização ${ }^{(9)}$.

Nos cuidados de reabilitação, as intervenções mais utilizadas foram: aspiração de secreções em circuito fechado (97\%); vibrações (93\%); I-EM (36\%), drenagem postural modificada (34\%); descompressão brusca (87\%); aberturas costais seletivas; expiração manual forçada (ambas com 80\%) e ventilação dirigida (51\%).

Num estudo prevalência pontual (em $47 \mathrm{UCl}^{\prime}$ s, a 230 doentes com VMI), Mackenzie et al recolheram dados relativos às técnicas de higiene brônquica. A aspiração de secreções foi a mais usada (96\%), sendo a aspiração em circuito fechado o método mais frequente $(68 \%)$. Outras técnicas de higienização brônquica apenas foram aplicadas a 84 doentes, nomeadamente: vibração (40\%); hiperinsuflação manual (29\%); percussão (24\%); drenagem postural / posicionamento (20\%) e outras técnicas, como a mobilização (18\%) ${ }^{(10)}$.

Neste caso, a persistência do compromisso da função respiratória, com hipoxémia refratária apesar da implementação de estratégias invasivas e terapêuticas adjuvantes como a ventilação protetora, a ECMO- VV e o PP (efetuado 12 vezes com uma duração entre 4 e 23 horas), ditou a decisão da aplicação do I-EM como técnica de limpeza das vias aéreas. Esta decisão foi ponderada pela equipa multidisciplinar, atendendo à gravidade do quadro clínico, risco de propagação de aerossóis, possíveis iatrogenias, sendo necessário 3 profissionais para a implementação de técnica. A programação do equipamento foi realizada pelo EEER, de acordo com os parâmetros ventilatórios do doente (pressões de in-exsuflação simétricas e semelhantes à pressão de plateau; tempos de in-exsuflação igualmente simétricos).

Em nenhuma sessão de CR em que se usou o I-EM foram registados eventos adversos (hemodinâmicos, ventilatório e remoção de dispositivos médicos). Apesar de atualmente não existir fundamentação científica que suporte este facto, é consensual que a I-EM no doente com VMI é uma técnica recente, segura e com aceitação crescente em UCl.
Pela análise dos dados verifica-se que nas intervenções em que foi utilizado o I-EM, houve melhoria de todos os outcomes, com maior impacto no volume inspiratório e compliance. Dados corroborados por Camillis et al (11) que mostraram que com a implementação de um protocolo de in-exsuflação mecânica, a quantidade de secreções aspiradas foi superior em comparação com a CR padrão, diminuição da resistência das vias aéreas e o aumento da compliance.

De igual modo, através da análise dos dados é possível denotar que as intervenções de $C R$, no período em ECMO- VV, em que foi utilizado I-EM houve melhoria de todos os outcomes, à excepção da $\mathrm{PaCO} 2$, que diminuiu ligeiramente. Ainda não existe evidência científica do uso do I-EM em doentes ventilados em suporte de ECMOVV. Contudo, Cork, Barrett \& Ntoumenopoulos (12), descrevem a eficácia da técnica de hiperinsuflação mecânica (através do ventilador) na promoção da higiene brônquica na pessoa com ECMO- VV.

Perante o exposto, é essencial que no futuro se desenvolvam estudos no âmbito da CR no doente COVID19 ventilado mecanicamente, atendendo que não existem protocolos publicados quanto à intensidade, frequência e duração das intervenções. Contudo, há evidência que sugere que intervenções estruturadas e individualizadas podem facilitar a recuperação da pessoa em situação crítica. Assim como, o EEER deve participar sempre na avaliação da condição física dos doentes na alta e no pós-alta ${ }^{(13)}$.

\section{CONCLUSÃO}

Com este estudo de caso, descrevemos as intervenções do ER mais utilizadas nas sessões de CR no cuidar do doente crítico com COVID-19 em contexto de VMI, com ECMO e a introdução do I-EM. Verificou-se que a implementação de técnicas de CR é possível, eficaz e contribui significativamente para a melhoria do estado de saúde da doente. Espera-se que este caso contribua para dar visibilidade à importância que a Enfermagem de Reabilitação poderá ter na diminuição das comorbilidades resultantes desta doença.

Este exercício reflexivo permitiu à equipa refletir acerca das possibilidades de melhoria na intervenção do EEER na CR na doente.

\section{AGRADECIMENTOS}

Agradecemos a todos os profissionais de saúde da $\mathrm{UCI}$ pela sua dedicação e empenho nesta fase de pandemia COVID-19. Agradecemos igualmente ao Núcleo de Investigação em Enfermagem do Centro Hospitalar, na pessoa do enfermeiro Ricardo Ferreira pelo apoio prestado na elaboração deste estudo de caso.

\section{REFERÊNCIAS BIBLIOGRÁFICAS}

1. Sociedade Portuguesa de Medicina Física e Reabilitação. Plano de Atuação na Reabilitação de Doentes Admitidos na Unidade de $\begin{array}{llll}\text { Cuidados Intensivos por } & \text { COVD-19 }\end{array}$ https://www.spci.pt/media/covid19/COVID19_Recomendacoes_ SPMFR_para_doentes_UCI.pdf

2. Organização Mundial de Saúde. Clinical management of severe 
acute respiratory infection (SARI) when COVID-19 disease is suspected. Interim guidance. 2020; 13 March (WHO/2019$\mathrm{nCoV} /$ Clinical/2020.4)

3. Lucchini A, De Felippis C. Pelucchi G. Grasselli G. Patroniti N, Castagna L, Et al. Application of prone position in hypoxaemic patients supported by veno-venous ECMO. Intensiv Crit Care Nurs. 2018; 48, 61-68.

4. Budgell, B. Guidelines to the writing of case studies. J Can Chiropr Assoc. 2008; 52(4): 199 - 204

5. Azevedo PM, Gomes BP. Efeitos da mobilização precoce na reabilitação funcional em doentes críticos: uma revisão sistemática. Rev Enferm Ref. 2015; Série IV (5): 129-138. Disponível em: doi.org/10.12707/RIV14035

6. Stiller K. Physiotherapy in Intensive Care An Updated Systematic Review. Chest J. 2013; september; 144 (3): 825-47.

7. Machado M. Bases da Fisiologia Respiratoria: Terapia Intensiva e Reabilitação. 2 ed. Guanabara Koogan; 2018.

8. Mendes JJ, Mergulhão P, Froes F, Paiva JA, Gouveia J. Recomendações da Sociedade Portuguesa de Cuidados Intensivos e Grupo de Infeção e Sépsis para a abordagem do COVID-19 em medicina intensiva. Rev Bras Ter Intensiva. [Internet]. $2020 \mathrm{Mar}$ [cited 2020 abril 16];32(1):2-10. Available from:: https://www.spci.pt/media/covid-
19/COVID_19_R_20200416.pdf

9. Mesa do Colégio da Especialidade de Enfermagem de Reabilitação. Orientações covid-MCEER: Cuidados de Enfermagem de Reabilitação para pessoas com COVID-19. Ordem dos Enfermeiros. [Internet]. 2020. Available from: https://www.ordemenfermeiros.pt/media/17940/mesa-docol\%C3\%A9gio-da-especialidade de-enfermagem-dereabilita\%C3\%A7\%C3\%A3o-orienta\%C3\%A7\%C3\%B5es-covid-19.pdf

10. Ntoumenopoulos, G. Rehabilitation during mechanical ventilation: Review of the recent literature. Intensiv Crit Care Nurs. 2015; 31(3): 125-132.

11. Camillis MLF, Savi A, Rosa RG, Figueiredo M, Wickert R, Borges LGA, et al. Effects of mechanical insufflation-exsufflation on airway mucus clearance among mechanically ventilated ICU subjects. Respir Care. 2018; 63(12):1471-1477.

12. Cork G., Barrett N,. Ntoumenopoulos G. Justification for Chest Physiotherapy during Ultra- Protective Lung Ventilation and Extra-Corporeal Membrane Oxygenation: A Case Study Physiother. Res. Int. 2014; August; 19: 126-28.

13. Mendes R, Nunes M. A importância da enfermagem de reabilitação nas unidades de cuidados intensivos portuguesas. Rev Port Enferm Reabil [Internet]. 2018; Dez. V1(2):8-13. 